\title{
Two Neural Models for fast Category Learning - Neural Associative Memories and the Restricted Coulomb Energy Model*
}

\author{
A. König, A. Korn, F. Quint; M. Glesner \\ Darmstadt University of Technology \\ Institute for Microelectronic Systems \\ Karlstrasse 15, D-6100 Darmstadt, Germany
}

\begin{abstract}
In this work we provide a qualitative evaluation and comparison of two models for fast category learning. The first model is the Neural Associative Memory (NAM) model [1] and the second the Restricted Coulomb Energy (RCE) model [2]. The latter is embedded in a commercial product. Both models provide iterative training procedures that select prototypes from the initial training set. Both models allow supervised learning of pattern categories separated by arbitrary complex boundaries. NAM generates a piecewise linear approximation of the class boundaries, RCE a pieceswise approximation with hyperspherical surfaces. Training is an incremental process, as prototypes are iteratively inserted or deleted from the network. Thus, the training process is much faster than for other comparable interpolative neural approaches, e.g. LVQ. No a priori knowledge of data distribution is necessary, as the correct amount of prototypes for each class is determined by the training procedures of NAM and RCE. Dynamic LVQ techniques also generate a sufficient number of prototypes for each class, but they do not optimize the number. All these vector quantizing approaches are very transparent and allow the easy interpretation of the weight vectors. RCE and NAM model both provide rapid training and recall, which makes them excellent candidates as classifiers in a generic inspection system for visual industrial quality control. In the context of the research project SIOB such a system is developed and implementations of NAM and RCE are tested as modules here. To assess the superior model qualitative and quantitative comparison is undertaken. Both models start with an initial empty set of prototypes. Driven by the objective to correctly classify the training patterns, prototypes are iteratively selected from these patterns. If the existing prototypes give correct classification no insertion takes place, else a new prototype is stored. There are two main differences between the models. The NAM conducts an unrestricted competition, whereas the $R C E$ has an radius of influence for each prototype. The NAM eliminates redundant prototypes, whereas RCE only adjusts the respective radii of influence. In the case of misclassification both models store a new prototype, RCE adjusts the according radii of influence. The unrestricted competition of the NAM can cause an overfitting to the training set. Thus, in this work the NAM model was extended introducing thresholds of similarity for training and recall. This approach was validated with data from the SIOB project. Based on this qualitative comparison the authors will in future work elaborate a quantitative comparison using SIOB feature data obtained from an inspection line. The interest of the authors is to obtain a rapid classification mechanism for the visual quality inspection system supporting rapid installation and change of objects.
\end{abstract}

\section{References}

[1] W. Pöchmüller, A. König, M. Glesner, "Iterative Data Reduction Algorithms and their Application to Binary Associative Networks", in Proceedings of the International Joint Conference on Neural Networks IJCNN'92, Vol. II, pp. 3 - 9, Beijing, 1992

[2] D. L. Reilly, L. N. Cooper, C. Elbaum, "A Neural Model for Category Learning", Biological Cybernetics, 45, pp. $35-41,1982$

\footnotetext{
*This work was accomplished within the scope of a research project for visual object inspection in industrial quality control under grant of the German Federal Ministry of Research and Development (BMFT) grant number 01 IN $110 \mathrm{~B} / 6$ (SIOB)

${ }^{\dagger} \mathrm{Dr}$. A. Korn and F. Quint are with Fraunhofer-Institut für Informations- und Datenverarbeitung IITB Karlsruhe
} 